\title{
INESC Porto Experimental SMART GRID: Enabling the Deployment of EV and DER
}

\author{
C. Gouveia, D. Rua, F. Ribeiro, C. L. Moreira, J. A. Peças Lopes \\ INESC TEC - INESC Technology and Science (formerly INESC Porto) \\ Porto, Portugal \\ cstg@inescporto.pt, drua@inesporto.pt, fasr@inescporto.pt, cmoreira@inescporto.pt, jpl@fe.up.pt
}

\begin{abstract}
The feasibility of the MicroGrid (MG) concept, as the pathway for integrating Electric Vehicles (EV) and other Distributed energy Resources (DER), has been the focus of several research projects around the world. However, developments have been mainly demonstrated through numerical simulation. Regarding effective smart grid deployment, strong effort is required in demonstration activities, addressing the feasibility of innovative control solutions and the need of specific communication requirements. Therefore, the main objective of this paper is to provide an integrated overview of the laboratorial infrastructure under development at INESC Porto, where it will be possible to conceptualize, implement and test the performance of new control and management concepts for Smart Grid cells. The laboratorial infrastructure integrates two experimental MG, including advanced prototypes for power conditioning units to be used in micro generation applications, batteries for energy storage and a fully controlled bidirectional power converter. Preliminary experimental results and organization of the infrastructure are presented.
\end{abstract}

Index Terms - Electric Vehicle, islanding operation, Microgeneration, MicroGrids, power engineering education, Smart Metering.

\section{INTRODUCTION}

The Smart Grid initiative aims to develop a reliable, costeffective and low carbon power system. To achieve these goals, the distribution system needs to be redesigned assuming high levels of Distributed Energy Resources (DER) that can be managed by clusters of Microgrids (MG) [1].

A MG is a highly flexible Low Voltage (LV) system, which can operate either interconnected (normal operating mode) or islanded from the Medium Voltage (MV) network (emergency operating mode). In order to be flexible and controllable, the MG resources are coordinated by the hierarchical management and control system shown in Fig.1, constituted by a network of local controllers headed by the MG Central Controller (MGCC).

The adoption of innovative control strategies at EV charging interface level, allows exploiting the EV flexibility, which can act both as a controllable load or a storage device, in the Vehicle-to-Grid (V2G) operation mode [2], [3]. When connected to the network, the control of the EV charging power can provide voltage and frequency grid support, enabling the increase in the amount of renewable based generation that can be safely integrated in the grid and enhancing the MG robustness when operating autonomously.

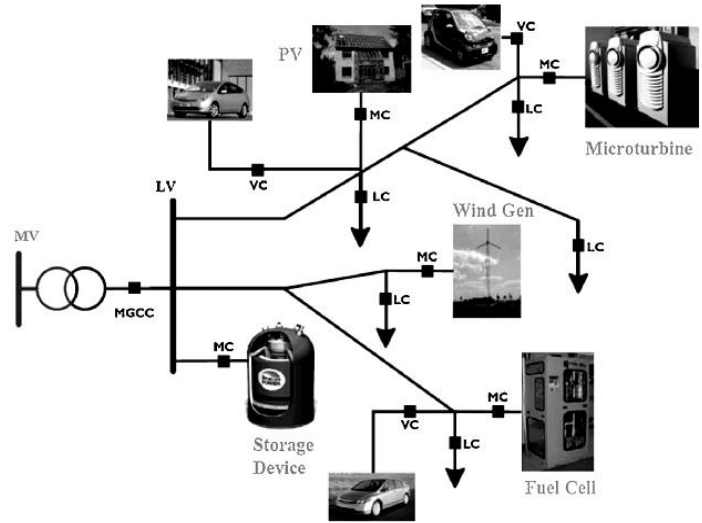

Figure 1.Basic Architecture of a Microgrid [2]

The feasibility of the MG concept integrating EV has also been the focus of several research projects around the world, which played a key role on the identification of adequate control strategies and architectures. However, experimental validation is required in order to effectively validate the developments in near real-world conditions. A review of worldwide experimental MG and pilots can be found in [4][5]. CERTS/AEP MG full-scale test facility has played a key role on the validation of the CERTS MG concept, namely in what respects the MG control architecture and protection system [6]. European laboratory facilities such as DEMOTEC or Labein Experimental Centre have also been involved in the validation of the European MG concept, as well as on the test and certification of new microgeneration technologies [5]. More recently, under the smart grid research initiatives, new pilots and laboratory infrastructures are being built, focused on the integration of $\mathrm{EV}$, demand side management strategies and smart metering technology testing [7]. 
The main objective of this paper is to present the laboratorial infrastructure under development at the INESC Porto facilities, where it will be possible to test the performance of new control and management concepts, where DER and EV are managed in an integrated manner within the MG concept. This paper provides a detailed description of the implemented smart grid architecture, along with several functionalities to be implemented in the different management and control levels.

\section{OVERVIEW OF INESC PORTO LABORATORIAL FACILITIES}

This laboratorial facility was conceived for developing advanced research activities that will allow an active integration of EV in the smart distribution grid control architecture [8]. An important outcome of this research was the identification of innovative control strategies for the renewable based microgeneration technologies, envisioning the development of new distribution grid support services [9]-[12].

\section{A. Electrical infrastructure}

The laboratorial architecture was envisioned to be fully open and flexible, with a scalable structure, in order to allow an individual and integrated testing of new concepts and control algorithms to be housed at the different smart grid hierarchical levels, as well as different communication architectures, technologies and protocols. Fig. 2 presents the laboratory electric and automation equipment, which includes: renewable based microgeneration $(3 \mathrm{~kW}$ wind micro-turbine emulator and $6 \mathrm{kWp}$ photovoltaic panels), storage (25 kWh capacity Flooded Lead-Acid (FLA) battery banks 128 Lithium battery cells for the EV charging prototype), a $54 \mathrm{~kW}$ resistive load bank and a plug-in electric vehicle, which is charged by a single-phase commercial home charger.

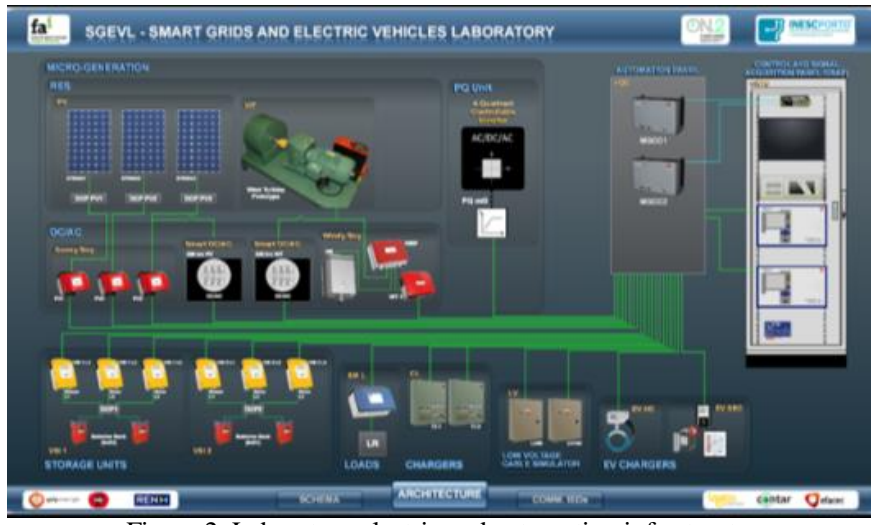

Figure 2. Laboratory electric and automation infrastructure.

The microgeneration units are coupled to the electric network through commercial inverters or through singlephase DC/AC bi-directional inverter prototypes developed in-house. These prototypes were developed in order integrate additional local control strategies. The laboratory is also equipped with a 4 quadrant $\mathrm{AC} / \mathrm{DC} / \mathrm{AC}$ inverter, which allows emulating the operating characteristics of controllable microgeneration technologies such as single-shaft microturbines or solid oxide fuel cells.
The FLA battery bank is connected to two three-phase groups of SMA Sunny Islands, constituting two isolated MG, supplying both three-phase and single-phase loads and micro-generation units.

In order to implement the developed smart charging and V2G strategies, a DC/AC bi-directional inverter prototype was also developed and connected to a lithium battery bank.

All devices are connected through an electric panel with six $400 \mathrm{~V}$ busbars, interconnected by switches and with thirty outputs connected to the laboratorial equipment. The configuration of the electric panel is very flexible, allowing the composition of different MG configurations. All feeders are commanded through contactors and can be connected to one of the three semi busbars that exist in the electric panel.

The laboratorial infrastructure implements a three-phase four wire LV system, with both single-phase and three-phase microgeneration and loads and single-phase EV chargers. The LV cables emulators (100A and 50A) enable the development of different network testing scenarios, considering the resistive nature of LV feeders. Different unbalanced scenarios can also be implemented, distributing the laboratory electric devices by the three-phases of the system.

The laboratory supervision and automation is carried out through a SCADA system. This system supports the laboratory operation, being responsible for the electrical network reconfiguration, protection and metering infrastructure. Each feeder and busbars is equipped with a dedicated metering device with voltage and current accuracy of $0.2 \%$. In addition to a large quantity of electrical values collected, the metering equipment provides a set of power quality indicators, such as voltage and current harmonics (up to the 25th order) and symmetric components. The devices communicate using a ModBus protocol over a RS-485 network.

\section{B. Communication and Control Architecture}

Similarly to the MG architecture presented in Fig. 1, an information and communication infrastructure was implemented overlaid to the electric configuration, constituting the MG management and control structure. As represented in Fig. 3, the management and control structure was implemented considering three levels, namely: the DM\&CS Distribution Management and Control System, which coordinates the operation of the distribution network, the Microgrid Central Controller (MGCC) that ensures the management and control of the MG and the lower control level constituted by smart meters (SM), associated to MG local controllers, namely: Microsource Controller (MC), Load Controller (LC) and Vehicle Controller (VC).

The SM acts as a gateway between the MGCC and the local controllers, having bi-directional communication capabilities. It is able to receive set-points from the MGCC and redirect them downstream to the local controllers and send metering data and other type of information upstream. The SM has also local processing capability, in order to integrate the owner's preferences regarding the participation on grid support services and remote management of load and generation. As shown in Fig. 4, a graphic user interface was de- 
veloped, concentrating the current state of the owner's load, microgeneration and $\mathrm{EV}$.

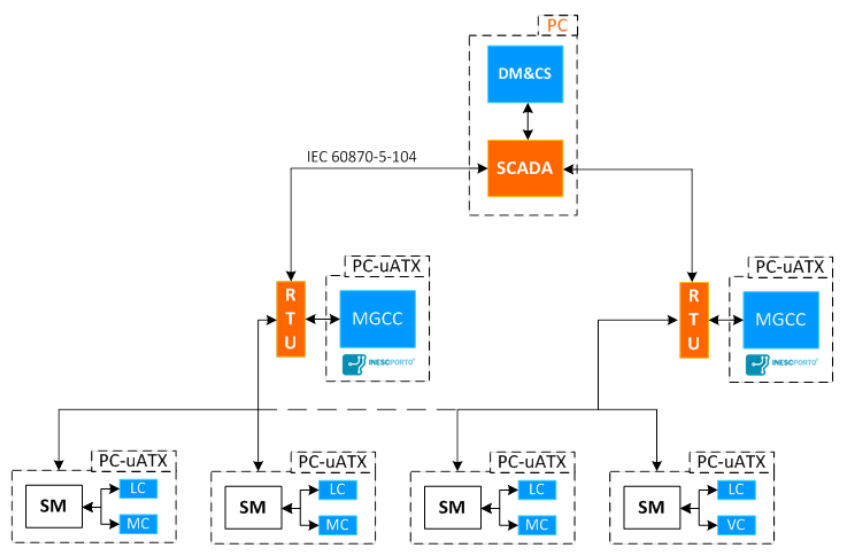

Figure 3. Laboratory management and control architecture.

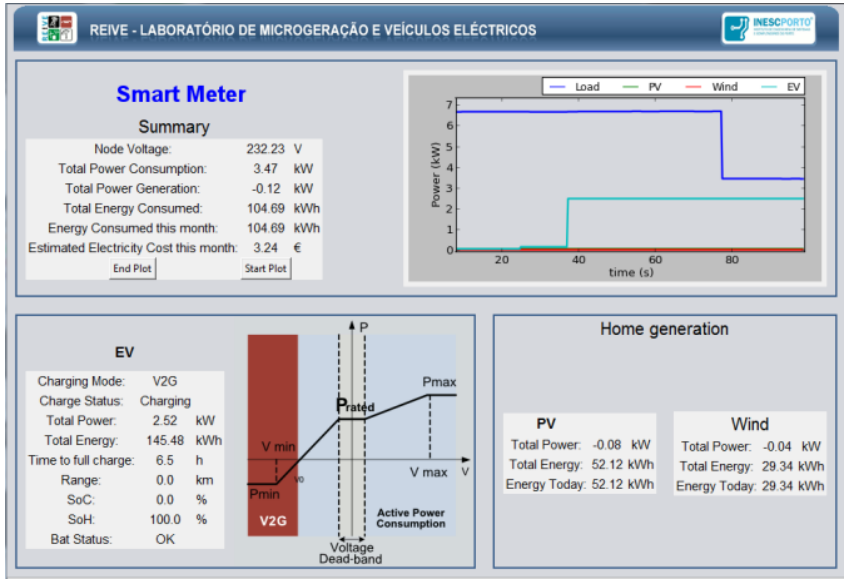

Figure 4. Smart Meter graphic user interface.

The MG high level management and control functionalities are housed in the MGCC. The information received from the smart meters namely: power generation, load, EV, responsive loads and power quality indicators are processed and aggregated according to the system operator needs. The information received is then used by local software modules, responsible for managing the $\mathrm{MG}$ technical operation, during normal and emergency conditions.

An Ethernet infrastructure is used to interconnect the different elements of the control structure, thus providing a communication network between the different control levels, while allowing an interaction with the SCADA system. This network it is also responsible for conveying the metered data from inverters, developed prototypes and the other laboratory equipment, through the use of specific protocol converters. A Medium Behavior Controller (MBC) was developed in order to allow the emulation of different communication technologies. The Ethernet network is used as a controllable communication medium where distributed MBCs are able to impose controlled bandwidth values to the different communication interfaces and define different communication profiles considering data packet delays and losses.

\section{MG CONTROL FUNCTIONALITIES}

The MG has two main operating modes: normal, when operating interconnected to the MV network, and emergency, when operating autonomously. In normal operating mode the MG main objective is to accommodate the power generation from RES or EV power consumption, while assuring adequate power quality levels for the MG consumers. When the MG operates islanded, the main operating objective is to maintain the stability of the system, ensuring frequency and voltage regulation and load following capabilities.

The hierarchical structure shown in Fig. 3 ensures the MG management and control, for both interconnected and islanded operation. The MGCC is responsible for the coordinated management of the $\mathrm{MG}$ resources, integrating a set of software modules responsible for the LV network monitoring, centralized voltage control, storage management and emergency power dispatch for secondary frequency regulation. As a result, the MGCC will issue a set of control signals (i.e. power, frequency and voltages set points or on/off commands) to the MG local controllers.

These controllers are active elements, providing voltage and frequency regulation, based in configurable droop characteristics and local metering data. This approach increases the $\mathrm{MG}$ robustness and reduces the burden over the $\mathrm{MG}$ communication infrastructure, which is particularly important during emergency operation.

\section{A. MG Voltage Control}

Quite often LV distribution networks suffer from a voltage rise effect, due to the connection of microgeneration units at the end of long feeders. This results from the low $\mathrm{X} / \mathrm{R}$ ratio that characterizes this type of network and from the reduced simultaneity between load and renewable generation, such as in the case of solar photovoltaic generators. Under certain conditions, significantly high voltage profiles in LV networks might lead to overvoltage tripping of microgeneration, thus limiting the amount of renewable based generation that can be integrated in the system without compromising power quality. In order to overcome this undesired limitation, it is necessary to develop control mechanisms at the microgeneration power electronic interfaces, in order to control the power injected in the LV network.

At the local control level, the MS injected power and the $\mathrm{EV}$ power consumption is controlled by droop control functionality, relating node voltage deviations with active power injections. The microgeneration active power-voltage droop characteristic is shown in Fig. 5. The general idea of local control rule is as follows: there is a voltage dead-band within which the system operator defines the reference power $\left(P_{r e f}\right)$ that can be injected in the grid. If the voltage rises above the dead-band, the local control autonomously starts reducing the injected power. On the contrary, if the voltage drops below the dead-band the local control allows a power increase, limited to the maximum power $\left(P_{\max }\right)$ that can be extracted from the primary source.

The EV active power-voltage droop characteristic adopted is shown in Fig. 6. Accordingly, the EV will increase its power consumption when the voltage rises to values outside 
the dead-band, increasing the total load of the MG. Similarly, when the MG voltage drops, the EV will decrease its power consumption or even inject power into the MG, by activating the so called $\mathrm{V} 2 \mathrm{G}$ feature.

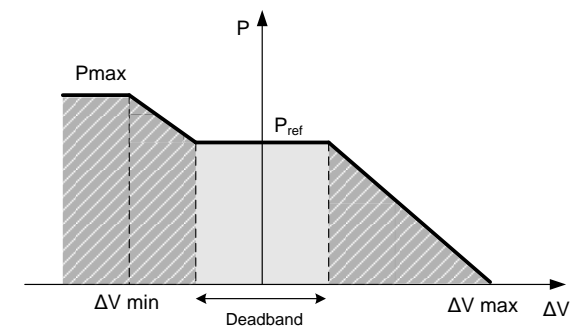

Figure 5. Microgeneration P-V droop characteristic.

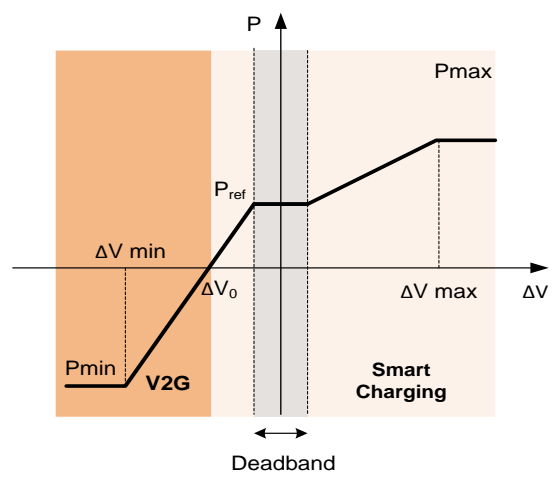

Figure 6. EV P-V droop characteristic.

At the higher control level, the MGCC has the responsibility for periodically defining the most adequate parameters of the droop function operating at each microgeneration unit.

\section{B. MG Frequency control}

When the MG disconnects from the main grid, due to a fault upstream or a planned outage, it is necessary to establish the MG voltage in magnitude and frequency. Therefore, the MG requires specific primary and secondary frequency control strategies, in order to maintain power balance between generation and loads and restore frequency to adequate values. The MG primary frequency control is ensured by the storage units grid-coupling devices, which are controlled through an active power-frequency droop characteristic. In islanded mode, power unbalance between generation and load is compensated by storage units, which will continue to inject/absorb power until the secondary frequency control corrects frequency to nominal values.

Similarly to the voltage control strategies presented in previous subsection, the MS and EV can also participate in the $\mathrm{MG}$ frequency regulation through droop functions, in order to decrease the solicitation from the storage units and improve the MG stability. When the power generation exceeds the load, the MG frequency will rise and the storage unit will absorb the power resulting from the unbalance. By adopting a P-V droop characteristic, the MS will decrease their power output and the EV will increase its power consumption. However, if the MG load exceeds power generation, the EV will decrease its power consumption or even inject power into the MG. In this case, the MS will only in- crease their power consumption if the MS injected power $\left(P_{r e f}\right)$ is lower than the maximum admissible power $\left(P_{\max }\right)$.

In order to implement the desired frequency and voltage control functionalities within the laboratory infrastructure several developments were required. The power electronic interfaces for PV panels, micro wind turbines and EV prototype were developed, considering the local control strategies presented in Fig. 5 and Fig. 6. The variation in the power injected by the PV panels is implemented by modifying the maximum power point tracking algorithm, thus temporally degrading the efficiency of the PV panels operating conditions [9]. The control of the micro wind turbines power injection was achieved through the use of a dump load, which allows the dissipation of the power surplus produced by the turbines [10]. The EV droop characteristics define the reference (dis)charging power to the DC-DC converter, which regulates the power supplied to or by the battery [11], [12].

\section{EXPERIMENTAL RESULTS}

In order to demonstrate the potential of the INESC Porto laboratorial facilities, the experimental MG topology represented in Fig. 7 was adopted in this research. The system includes two PV strings connected to a DC/AC solar power converter prototype, a wind-turbine emulator, an EV charger prototype and a $27 \mathrm{~kW}$ resistive bank. The MG node is interconnected to the main grid through a $100 \mathrm{~A}$ LV cable emulator, which has a $0.6 \Omega$ resistance. The MG control strategies presented in section III were tested for both interconnected and islanded operation. The communication architecture, illustrated in Fig. 8, was used to support the data control exchanged during the different phases of the scenarios considered.

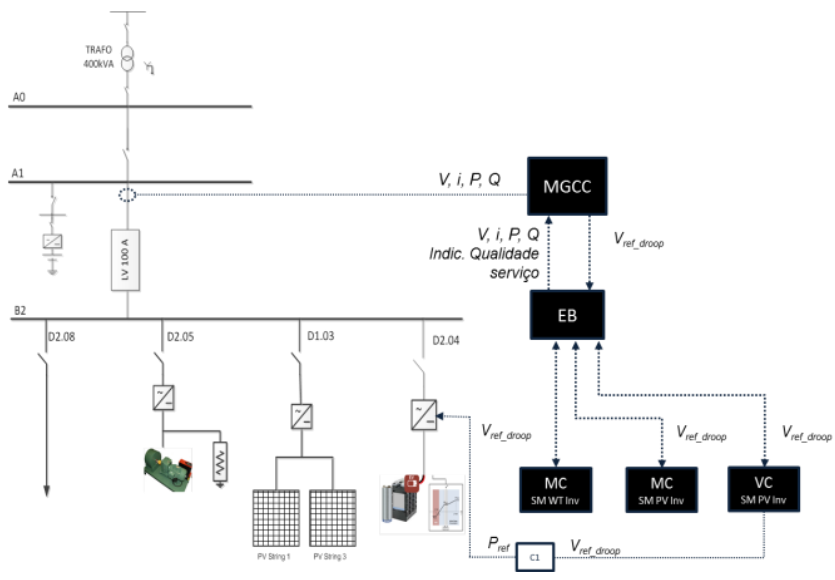

Figure 7. MG experimental test system. 


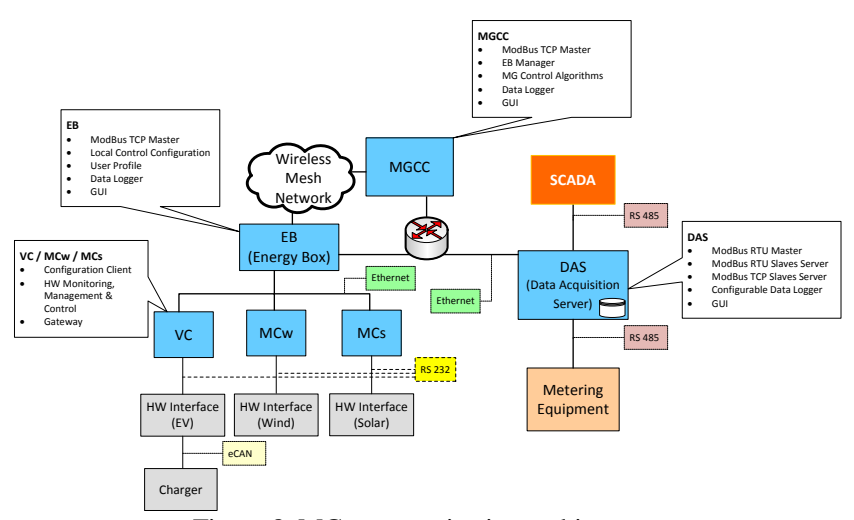

Figure 8. MG communication architecture.

\section{A. Interconnected Mode}

The main objective of the MG interconnected scenario is to evaluate the performance of the $\mathrm{P}-\mathrm{V}$ droop control incorporated at the microgeneration units and at the EV power electronic converters (single-phase units) and its remote parameterization through the MGCC. Two different tests were performed, in order to simulate two extreme cases at different hours of the day. The first scenario considers high penetration of RES based generation in low load conditions. The second test considers no power generation with high load scenarios, simulating the night peak of load diagram.

Fig. 9 provides an overview of the results obtained for the first test. At $\mathrm{t}=104 \mathrm{~s}$, the micro-wind generator power production increased to $1.8 \mathrm{~kW}$ approximately. As shown in Fig. 10 , the voltage in phase A of node 2 suffers a significant increase. At $\mathrm{t}=167 \mathrm{~s}$, the PV panel starts to inject power into the grid, causing a voltage increase from $240 \mathrm{~V}$ to $247.2 \mathrm{~V}$. As a result of the actual phase voltage, the MGCC remotely sends to the EB a command to enable the droop control functionality in the micro wind turbine emulator at $t=230 \mathrm{~s}$, making it possible to observe a reduction on the active power injected by this unit, as well as the subsequent reduction in the phase voltage. At $\mathrm{t}=270 \mathrm{~s}$, the droop control functionality in the PV panel power electronic interface was also activated. Since both the units are injecting the same power and have the same droop parameters, they share the active power reduction equally.

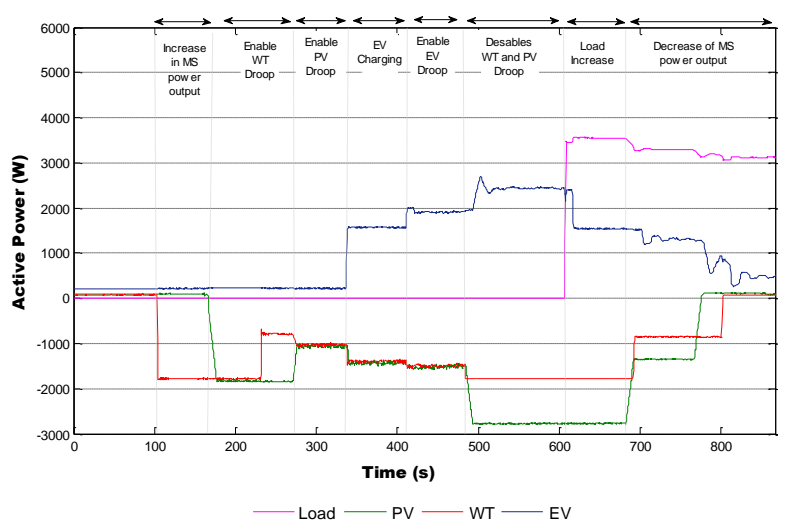

Figure 9. Main results of the MG interconnected scenario - test 1.

At $\mathrm{t}=330 \mathrm{~s}$, the EV charger prototype is connected to the MG and starts charging with a reference power of $1.5 \mathrm{~kW}$.
Since the MG load increases and the voltage decreased, the microgeneration units increase their power output.

In order to show the effectiveness of the MG control strategy, the EV P-V droop was activated at $\mathrm{t}=410 \mathrm{~s}$ and the microgeneration units voltage droop was disabled at $t=480 \mathrm{~s}$. As shown in Fig. 9 and Fig. 10, the microgeneration power output has increased, raising the MG voltage. As a consequence the $\mathrm{EV}$ increases its power consumption to approximately $2.5 \mathrm{~kW}$, maintaining the voltage at $242 \mathrm{~V}$. At $\mathrm{t}=600 \mathrm{~s}$ a $3 \mathrm{~kW}$ load was connected and the voltage dropped below $235 \mathrm{~V}$. Since this voltage is within the EV droop dead-band, the EV decreases the charging power to the reference value $(1.5 \mathrm{~kW})$. At $\mathrm{t}=680 \mathrm{~s}$ the power output from the microgeneration units started to decrease, simulating the end of the day. As a consequence the voltage dropped below $225 \mathrm{~V}$, surpassing the voltage dead-band of the EV voltage droop, which consequently decreased its charging power.

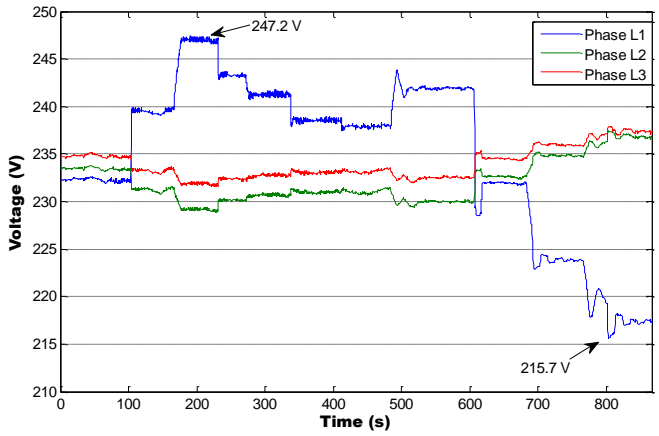

Figure 10. Voltage at node 2 for the MG interconnected scenario-test 1.

Fig. 11 shows the results obtained for the second test. In the beginning of the experiment the EV was already charging. At $\mathrm{t}=60 \mathrm{~s}$ the $\mathrm{MG}$ load increased, taking the $\mathrm{MG}$ voltage to fall below $220 \mathrm{~V}$. Based on the metered voltage, the MGCC activates the EV charger prototype voltage droop control, and the EV decreases its power consumption, while maintaining the voltage at $220 \mathrm{~V}$. At $\mathrm{t}=140 \mathrm{~s}$ the commercial EV starts to charge, increasing the MG load and decreasing the voltage below $215 \mathrm{~V}$. According to the droop characteristic of the charger the EV prototype starts to inject power into the grid, activating the V2G mode.

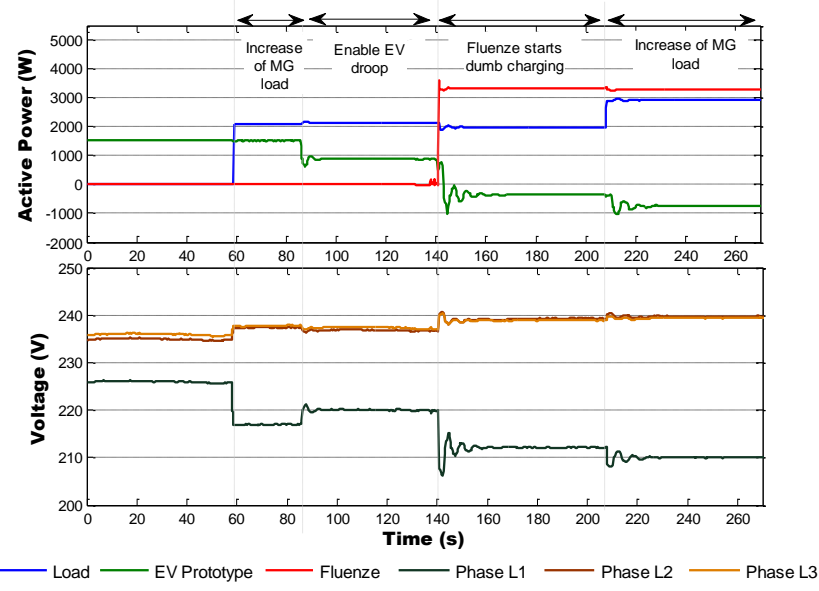

Figure 11. Main results of the MG interconnected scenario - test 2. 


\section{B. Islanded Mode}

In the beginning of the experiment the MG was connected to the main grid, importing approximately $12 \mathrm{~kW}$ from the upstream network. The EV prototype is charging and the microgeneration units were maintained disconnected in order to simulate a worst case scenario. One of the three-phase SMA Sunny Island group was synchronized with the main grid and it was charging the battery banks.

Fig. 12 provides an overview of the MG state during the experiment, regarding load, generation, EV and the grid. The SMA Sunny Island is disconnected from the main grid at $\mathrm{t}=$ 33 s to simulate an unplanned islanding event.

Fig. 13 compares the MG frequency response and the EV power output after the islanding. The MG frequency decreases bellow $48.6 \mathrm{~Hz}$. Since the zero-crossing frequency of the EV droop characteristic dropped below $49.5 \mathrm{~Hz}$, the EV inverted the power flow and started to inject power into the grid. The Sunny Island secondary control recovers the frequency to $50 \mathrm{~Hz}$ in about $3 \mathrm{~s}$, and the EV returns to its reference charging power $(1.5 \mathrm{~kW})$.

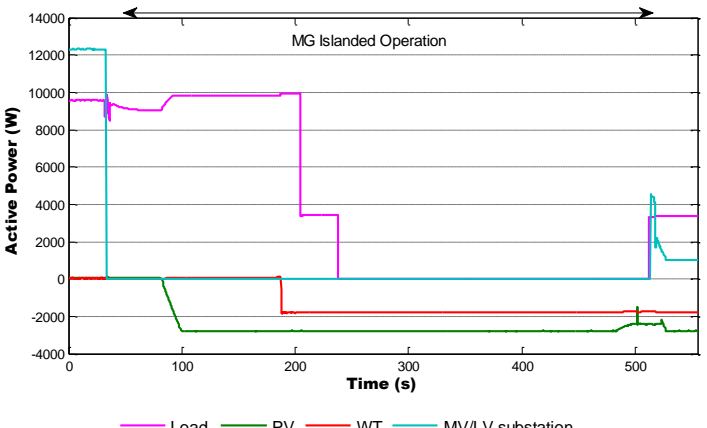

Figure 12. Main results of the MG islanded scenario.

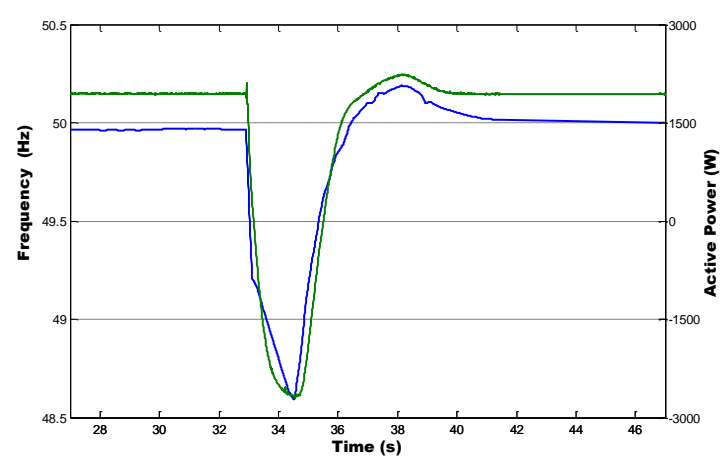

Figure 13. MG frequency and EV active power response during the unplanned islanding event.

At $\mathrm{t}=100 \mathrm{~s}$ the microgeneration starts to inject power into the grid and the load starts to decrease at $\mathrm{t}=200 \mathrm{~s}$. Since the MG generation exceeds the load, the Sunny island inverters start to charge the FLA battery bank. After some time, the batteries are fully charged and the frequency starts to increase. As shown in Fig.14, for $\mathrm{t}=324 \mathrm{~s}$ the $\mathrm{MG}$ frequency surpassed the EV frequency dead-band $(50.1 \mathrm{~Hz})$. Therefore, the EV increased its charging power to its maximum $(3 \mathrm{~kW})$ due the operation of the frequency droop control. At $t=500$ s the Sunny Islands start the resynchronization process and the $\mathrm{MG}$ is reconnected at $\mathrm{t}=510 \mathrm{~s}$.

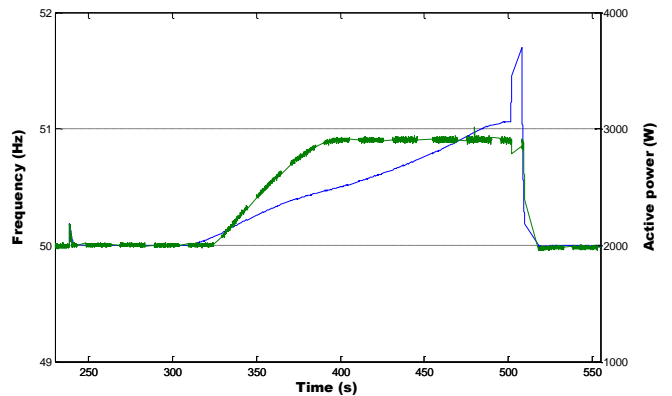

Figure 14. MG frequency and EV active power response in islanded mode, due to increase

V. CONCLUSIONS

The industrial development of smart grid related products and technologies are growing, enhancing the importance of experimental demonstrations which take into consideration real world conditions. The development of laboratorial facilities, such as the one described in this paper, plays an important role in the consolidation of innovative solutions that are key for a successful development of the smart grid paradigm, developing new prototypes of advanced interfaces for $\mathrm{EV}$ and microgeneration units. The results shown in this paper reinforce the fact that the coordination between the MG centralized and local control strategies, provides to system operators additional resources to deal with the crescent integration of DER resources and EV. The future work of this project is focused on the development of new software modules for the MG controllers, which will work in coordination with the devices installed in field (loads, EV, $\mu \mathrm{G}$, storage devices).

\section{REFERENCES}

[1] J R.H. Lasseter, "Smart Distribution: Coupled Microgrids," Proceedings of the IEEE, vol.99, no.6, pp.1074-1082, June 2011.

[2] J.A. Peças Lopes, Silvan A. Polenz, C.L. Moreira, Rachid Cherkaoui, "Identification of control and management strategies for LV unbalanced microgrids with plugged-in electric vehicles", Electric Power Systems Research, vol. 80, Issue 8, pp. 898-906, August 2010,

[3] J.A.P. Lopes, F. J. Soares, P. M. R. Almeida, "Integration of Electric Vehicles in the Electric Power System," Proceedings of the IEEE, vol.99, no.1, pp.168-183, Jan. 2011.

[4] N. Hatziargyriou, H. Asano, R. Iravani, C. Marnay, "Microgrids", IEEE Power and Energy Magazine, vol.5, no.4, pp.78-94, 2007.

[5] M. Barnes, J. Kondoh, H. Asano, J. Oyarzabal, G. Ventakaramanan, R. Lasseter, N. Hatziargyriou, T. Green, "Real-World MicroGrids-An Overview," IEEE International Conference on System of Systems Engineering, pp.1-8, 16-18 April 2007.

[6] CERTS Microgrid Test Bed Demonstration with American Electric Power, "CERTS Phase III-2011 Planned activities", Project presentation, 2011. Available at: http://certs.lbl.gov/certs-derkey-mgtb.html (Last consulted on Jan. 2013).

[7] KEMA DNV, "Smart Grid Projects", Report. Available at: http://www.dnvkema.com/innovations/smart-grids (Last consulted on Jan. 2013).

[8] Project REIVE - Smart Grids with Electric Vehicles [Online]: http://reive.inescporto.pt/en (Last consulted on Jan. 2013).

[9] J. Rodrigues F. O. Resende, C. L. Moreira, "Using Photovoltaic Systems to Improve Voltage Control in Low Voltage Networks", ISGT2012 - Third IEEE PES Innovative Smart Grid Technologies Europe Conference, Berlin, Germany, Ocotber, 2012.

[10] J. Rodrigues F. O. Resende, C. L. Moreira, "Contribution of PMSG based Small Wind Generation Systems to Provide Voltage Control in 
Low Voltage Networks, ISGT 2011 - IEEE PES ISGT 2011 Europe, Manchester, UK, 2011

[11] R. Ferreira, L. M. Miranda, R.E. Araujo, J. Peças Lopes, A new BiDirectional Charger for Vehicle-to-Grid Integration, IEEE PES ISGT 2011 Europe, Manchester, UK, Dezembro, 2011.
[12] D. Varajão, R.E.Araujo, C. Moreira, J. Peças Lopes, Impact of Phaseshift Modulation on the Performance of a Single-stage Bidirectional Electric Vehicle Charger, Proceedings of the 38th Annual Conference on IEEE Industrial Electronics Society IECON12, pp.5215-5220, Montreal, Canada, Outubro, 2012.

(C) 2013 IEEE. Personal use of this material is permitted. Permission from IEEE must be obtained for all other uses, in any current or future media, including reprinting/republishing this material for advertising or promotional purposes, creating new collective works, for resale or redistribution to servers or lists, or reuse of any copyrighted component of this work in other works. 